\title{
Chinese Word Segmentation based on Maximum Matching and Word Binding Force
}

\author{
Pak-kwong Wong and Chorkin Chan \\ Department of Computer Science \\ The University of Hong Kong \\ Pokfulam Road \\ Hong Kong \\ pkwong@cs.hku.hk and cchan@es.hku.hk
}

\begin{abstract}
A Chinese word segmentation algorithm based on forward maximum matching and word binding force is proposed in this paper. 'This algorithm plays a koy role in post-processing the output of a character or speech recognizer in determining the proper word sequence corresponding to an input line of character images or a speceh waveform. 'To support this algorithm, a text corpus of over 63 millions characters is cmployed to enrich an 80,000-words lexicon in terms of its word entries and word binding forces. As it stands now, given an input line of text, the word segmentor can process on the average 210,000 charactors per second when running on an IBM R.ISC System/6000 $3 \mathrm{~B}^{\mathrm{T} T}$ workstation with a correct word identification rate of $99.74 \%$.
\end{abstract}

\section{Introduction}

A language model as a post-processor is essential to a recognizer of spech or characters in order to determine the appropriate word sequence and hence the semantics of an input line of text or utterance. It is well known that an $\mathrm{N}$-gram statistics language model is just as effective as, but much more efficient than, a syntactic/semantic analyser in detcrmining the correct word sequence. $\Lambda$ necessary condition to successful collection of N-gram statistics is the existence of a comprohensive lexicon and a large text corpus. The latter must be lexically analysed in order to identify all the words, from which, N-gram statistics can be derived.

About 5,000 characters are being used in modern Chinese and they are the building blocks of all words. Almost every character is a word and most words are of onc or two character's long but, there are also abundant words longer than two characters. Before it, is segmented into words, a line of text is just a sequence of characters and there are numerous word segmentation alternatives. Usu- ally, all but one of these alternatives are syntaktically and/or semantically incorrect. This is the case because unlike toxts in English, Chinese texts have no word markers. A first step towards building a language model based on $\mathrm{N}$-gram statistics is to develop an efficient lexical analyser to iclentify all the words in the corpus.

Word segmontation algorithms belong to one of two types in gencral, vi\%, the structural (Wang ot al., 1991) and the statistical type (Lua, 1990)(Tua and Gan, 1994)(Sproat and Shih, 1990) respectively. A structural algorithm resolves segmentiation ambiguities by examining the structural rolationships between words, while a statistical algorithm compares the nsage froquencies of the words and their ordered combinations instead. Both appproaches have serious limitations.

\section{Maximum Matching Method for Segmentation}

Maximum matching (Liu et al., 1994) is one of the most popular structural segmentation algorithms for Chinese texts. 'This mothod favours long words and is a greody algorithm by design, hence, suboptimal. Segrnentation rnay start from either ond of the line without any difference in segmentiation results. In this paper, the forward direction is adopted. The major advantage of maximum matching is its efficiency while its segmentation accuracy can be expected to lie around $95 \%$.

\section{Word Frequency Method for Segmentation}

In this statistical approach in terms of word frequencies, a lexicon needs not only a rich repertoire of word entries, but also the usage frequency of cach word. To segment a line of text, each possible segmentation altemative is evaluated according to the product of the word frequencies of the words segmented. 'The word sequence with the highest frequency product is accepted as correct. This method is simple but its accuracy depends heavily on the accuracy of the usage frequencies. The usage frequency of a word differs greatly from 
one type of documents to another, say, a passage of world news as against a technical report. Since there are tens of thousands of words actively useel, one needs a gigantic collection of texts to make an accurate estimate, but by then, the estimate is just an average and it may not be suitable for any type of doctment at all. In other words, the variance of such an estimate is too groat making the estimate useless.

\section{The Lexicon}

Most Chineso linguists aceept the definition of a word as the minimum unit that is semantically complete and can be put together as building blocks to form a sentence. Mowever, in Chinese, words can be mited to form componnel words, and they in turn, can combine furthes to form yed higher ordered componmel words. As a matter of fact, componel words are extremely common and they exist in large numbers. It is impossible to include all compound words into the lexicon but just to keep those which are frequently used and have the word componentis mited closely. A lexicon was acequired from the Institule of Information Science, Academia Sinica in Taiwan. There are 78410 word entries in this lexicon, each associated with a usatge frequency. $\Lambda$ corpuss of over 63 million characters of news lines was acpunired from China. Due to enltural clifferences of the two societies, there are many words encombered in the corpus but not in the lexicon. The latter must therefore bo enriched before it can be applied to perform the lexical analysis. The first step towards this end is to morgo a lexicon publishod in China into this one, increasing; the number of word entries to 85,855 .

\section{The Proposed Word Segmentation Algorithm}

The proposed algorithm of this paper makes use of a forward maxirmm matching stratcegy to identify words. In this respect, this algorithm is a structural approach. Holer this strategy, errors anc: usually associatied with single-character words. If the first charactor of a line is iclentified as a singlecharacter word, what it means is that there is no multi-character word entry in the lexicon that start: with such a character. In that case, there is not much one can do about it. On the other hand, when a character is identified as a single-character: word $\beta$ following another word $\alpha$ in the line, one cannot help wondering whether the sole chatrater composing $\beta$ should not be combined with the suffix of $\alpha$ to form another word instead, oven il that means changing $\alpha$ into a shorter word. In that case, every possible word seguence alternative corresponding to the sub-sequence of characters from $\alpha$ and $\beta$ together will be evaluated according to the product of its constituent word binding foress.
The binding force of a word is a measure of how strongly the elaracters composing the word are bomud together as a single mint. T'his force is often equated to the usage frepuency of the word In this respect, the proposed algorithm is a stiatistical approach. It is as efficient as the maximmm matching methed because word binding foress and utilized only in exceptional cases. However, much of the word ambiguitios are eliminated, leading to a very high word identification accuracy. Segmentation exrors associated with multi-charactex words can bo reduced by ardeling or deleting words to or from the lexicon as woll as arljusting word bincling forces.

\section{Structure of the Lexicon}

Worch in the lexicon are divided into 5 groups according to word lengths. They correspond to words of $1,2,3,4$, and more than 4 chamacters with group sizes equal to $7025,53532,12939$, 11269 , and 1090 respectively. Since most of the time spent in analyzing a line of text is in finding; a match anong the lexicon entries, a clever organization of the lexicon speeds up the searching process tremendously. Most Chinese words are of one or two characters only. Searching for longer words before shorter ones as practised in maximomn matching moans spending a groat doal of time searching for non-existent targets. 'Lo overcome this problem, the following measures are taken to organize the lexicon for fast someth:

- All single character words auce stored in a table of 32768 bins. Since the intemal code of a character takes 2 bytes, bits 1-15 are used as the bin arderess for the word.

- All 2-character words are stored in a separate table of 65536 bins. 'The two low order bytes of the two characters are used as a short integer for bin address. Should there be other words contesting for the same bin, they are kept, in a linked list.

- Any 3-character word is split into a 2chancter prolix and a 1-chanacter sullix. 'I'he prefix will be stored in the bin table for 2character words with clear indication of its prefix status. The suffix will be stored in the bin table for 1-charactor words, again, with char indication of its suffix status. All duplicate contries are combined, i.e., if $\alpha x$ is a word as woll as a suflix, the two entries are combined into (ne with an indication that it can serve as a word as well as a suffix.

- Any 4-character word is divided up into a 2character prefix and a 2-character suffix, both stored in the bin tiable for 2-character words, with clear indications of their respective stiatus. Wach profix points to a linked list of associated suffixes. 
- Any word longer than 4 characters will be divided into a 2-character prefix, a 2-character infix and a suffix. The prefix and the infix are stored in the bin table for 2-character words, with clear indications of their status. Each prefix points to a linked list of associated infixes and each infix in turn, points to a linked list of associated suffixes.

Maximum matching segmentation of a sequence of characters "...abcdefghij..." at the character "a" starts with matching "ab" against the 2-character words table. If no match is found, then, "a" is assumed a 1-character word and maximum matching moves on to " $b$ ". If a matich is found, thon, "ab" is investigated to see if it call be a prefix. If it cannot, then "ab" is a 2-character word and maximum matching moves on to "c". If it can, then one examines if it can be associated with an infix. If it can, then one examines if "cd" can be an infix associated with "ab". If the answer is negative, then the possibility of "abed" being a word is considered. If that fails again, then "c" in the table of 1-character words is examined to sec if it can be a suffix. If it can, then "abc" will be examined to see if can be a word by scarching the 1-character suffix linked list pointed at by "ab". Otherwise, one has to accept that "ab" is a 2-character word and moves on to start matching at "c". If "cd" can be an infix preceded by "ab", the linked list pointed at by "cd" as an infix will be searched for the longest possible suffix to combine with "abcd" as its prefix. If no match can be found, then one has to give up "cd" as an infix to "ab".

\section{Training of the System}

Despite the fact that the lexicon acquired from Taiwan has becn augmented with words from another lexicon developed in China, when it is applied to segment 1.2 million character news passages in blocks of 10,000 characters each randomly selected over the text corpus, an average word segmentation error rate $(\mu)$ of $2.51 \%$ was found with a standard deviation $(\sigma)$ of $0.57 \%$, mostly caused by uncommon words not included in the enriched lexicon. Then it is decided that the lexicon should be further enriched with now words and adjusted word binding forces over a number of gencrations. In generation $i, n$ new blocks of text are picked randomly from the corpus and words segmented using the lexicon enriched in the previous generation. This process will stop when $\mu$ levels off over several generations. The $100(1-\alpha) \%$ confidence interval of $\mu$ in generation $i$ is $\pm t_{0.5 \alpha, n-1} \sigma / \sqrt{n}$ where $\sigma$ is the standard deviation of crror rates in generation $i-1$, and $n$ is the number of blocks to be segmented in generation $i . t_{0.5 \alpha, n-1}$ is the density function of $(0.5 \alpha, n-1)$ degrees of free$\operatorname{dom}($ Devore, 1991). Throughout the experiments below, $n$ is always chosen to be 20 so that the $90 \%$ confidence interval (i.e., $\alpha=0.1$ ) of $\mu$ is about $\pm 0.23 \%$.

\section{Experimental Results}

The lexicon has been updated over six generations after being applied to word segment 1.2 million characters. The vocabulary increases from 85855 words to 87326 words. The segmentation error rates over seven generations of the training process are shown in the table below:

\begin{tabular}{|c|c|c|}
\hline \multirow{2}{*}{$\begin{array}{c}\text { Texicon } \\
\text { Generation } \\
\text { Number }\end{array}$} & \multicolumn{2}{|c|}{$\begin{array}{l}\text { Error Rate } \mu \text { over a text } \\
\text { of } 200,000 \text { Characters }\end{array}$} \\
\hline & Max. Mat. & $\begin{array}{l}\text { Max. Mat. \& } \\
\text { Word Bind. Force }\end{array}$ \\
\hline 0 & $5.71 \%$ & $2.32 \%$ \\
\hline 1 & $5.20 \%$ & $2.16 \%$ \\
\hline 2 & $4.66 \%$ & $1.88 \%$ \\
\hline 3 & $4.98 \%$ & $1.62 \%$ \\
\hline 4 & $2.60 \%$ & $0.43 \%$ \\
\hline 5 & $2.47 \%$ & $0 . \overline{30 \%}$ \\
\hline 6 & $2.44 \%$ & $0 . \overline{2} 6 \%$ \\
\hline
\end{tabular}

Most of these errors occur in proper nouns not included in the lexicon. They are hard to avoid unloss they become popular enough to be added to the lexicon. The CPU time used for segmenting a text of $1,200,000$ characters is 5.7 seconds on an IBM RiISC, System/6000 3BT computer.

\section{Conclusion}

Lexical analysis is a basic process of analyzing and understanding a language. The proposed algorithm provides a highly accurate and highly efficient way for word segmentation of Chinese texts. Due to cultural differences, the same language used in differcnt geographical regions and different applications can be quite different causing problems in lexical analysis. However, by introducing new words into and adjusting word binding forces in the lexicon, such difficulties can be greatly mitigated.

This word segmentor will be applicd to word segment the entire corpus of 63 million characters before N-gram statistics will be collected for post processing recognizer outputs.

\section{References}

Jay L. Devore. 1991. Probability and Statistics for Engineering and Sciences. Duxbury Press, pages $272 \cdot 276$.

Yuan Jiu, Qiang Tan, and Kun Xu Shen. 1994. The Word Segmentation Rules and Automatic Word Segmentation Methods for Chinese Information Processing (in Chinesc). Qing Hua University Press and Guang $X i$ Science and Technology Press, page 36. 
Kim-Ieng Lua and Kok-Wee Gan. 1994. An Application of Information Theory in Chinese Word Segmentation. Compuler Processing of Chinese and Oriental Languages, Vol. 8, No. 1, pages $115 \quad 123$, June.

K.T. Ina. 1990. From Character to Word $\cdots$ An Application of Information Theory. Computer Processing of Chinese and Oriental Languages, Vol. 4, No. 4, pages 304 313, March.

Liang-Jyh Wang, Tzusheng l'ei, Wei-Chuan li, and Lih-Ching R. Huang. 1991. A Parsing Method for Identifying Words in Mandarin Chinese Sentences. In Processings of toblh Inlernational Joind Conference on Artificial Intelligence, pages 1018 1023, Darling Ilarbour, Sydnoy, Australia, 24-30) August.

Richard Sproat and Chilin Shih. 1990. A Statistical Mothod for Finding. Word Boundaries in Chinese lext. Computer Processing of Chinese and Oriental languages, Vol. 4, No. 4, pagges 336-349, March. 